\title{
SPECTRAL CONTINUITY FOR OPERATOR MATRICES
}

\author{
SLAVISǍ V. DJORDJEVIĆ \\ University of Niš, Faculty of Philosophy, Department of Mathematics, Cirila and Metodija 2, \\ 18000 Niš, Yugoslavia \\ e-mail:slavdj@archimed.filfak.ni.ac.yu \\ and YOUNG MIN HAN \\ Department of Mathematics, Sungkyunkwan University, Suwon 440-746, Korea \\ e-mail:ymhan@math.skku.ac.kr
}

\section{(Received 21 March, 2000)}

\begin{abstract}
In this paper we prove that if $M_{C}=\left(\begin{array}{cc}A & C \\ 0 & B\end{array}\right)$ is a $2 \times 2$ upper triangular operator matrix on the Hilbert space $H \oplus K$ and if $\sigma(A) \cap \sigma(B)=\emptyset$, then $\sigma$ is continuous at $A$ and $B$ if and only if $\sigma$ is continuous at $M_{C}$, for every $C \in B(K, H)$.

1991 Mathematics Subject Classification. Primary 47A50, 47A53.
\end{abstract}

1. Introduction. Throughout this note let $H$ and $K$ be Hilbert spaces, let $B(H, K)$ denote the set of bounded linear operators from $H$ to $K$, and abbreviate $B(H, H)$ to $B(H)$. If $T \in B(H)$ write $N(T)$ and $R(T)$ for the null space and range of $T ; \alpha(T)=\operatorname{dim} N(T) ; \beta(T)=\operatorname{dim} N\left(T^{*}\right) ; \sigma(T)$ is the spectrum of $T ; \sigma_{a}(T)$ is the approximate point spectrum of $T ; \sigma_{d}(T)$ is the defect spectrum of $T ; \pi_{0}(T)$ is the set of eigenvalues of $T ; \pi_{00}(T)$ is the set of isolated points of $\sigma(T)$ that are eigenvalues of finite multiplicity. An operator $T \in B(H)$ is called left semi-Fredholm if it has closed range with finite dimensional null space and right semi-Fredholm if it has closed range with its range of finite co-dimension. If $T$ is both left semi- and right semi-Fredholm, we call it Fredholm. The index of a left semi- and right semi-Fredholm operator $T \in B(H)$ is given by

$$
i(T)=\alpha(T)-\beta(T) .
$$

The essential spectrum $\sigma_{e}(T)$, the Weyl spectrum $\omega(T)$ and the Browder spectrum $\sigma_{b}(T)$ of $T \in B(H)$ are defined in [5] and [6] as follows:

$$
\begin{aligned}
\sigma_{e}(T) & =\{\lambda \in \mathbb{C}: T-\lambda I \text { is not Fredholm }\} \\
\omega(T) & =\{\lambda \in \mathbb{C}: T-\lambda I \text { is not Weyl }\} \\
\sigma_{b}(T) & =\{\lambda \in \mathbb{C}: T-\lambda I \text { is not Browder }\} .
\end{aligned}
$$

Evidently

$$
\sigma_{e}(T) \subseteq \omega(T) \subseteq \sigma_{b}(T)=\sigma_{e}(T) \cup \operatorname{acc} \sigma(T),
$$

where we write $\operatorname{acc} G$ for the accumulation points of $G \subseteq \mathbb{C}$. We say that Weyl's theorem holds for $T \in B(H)$ if there is equality 


$$
\sigma(T) \backslash \omega(T)=\pi_{00}(T),
$$

and that Browder's theorem holds for $T \in B(H)$ if there is equality

$$
\omega(T)=\sigma_{b}(T) .
$$

If $\left(K_{n}\right)$ is a sequence of compact subsets of $\mathbb{C}$, then by the definition, its limit inferior is $\lim \inf K_{n}=\left\{\lambda \in \mathbb{C}:\right.$ there are $\lambda_{n} \in K_{n}$ with $\left.\lambda_{n} \rightarrow \lambda\right\}$ and its limit superior is $\lim \sup K_{n}=\left\{\lambda \in \mathbb{C}\right.$ : there are $\lambda_{n_{k}} \in K_{n_{k}}$ with $\left.\lambda_{n_{k}} \rightarrow \lambda\right\}$. If $\lim \inf K_{n}=\lim \sup K_{n}$, then $\lim K_{n}$ is defined by this common limit. A mapping $f$, defined on $B(H)$, whose values are compact subsets of $\mathbb{C}$, is said to be upper (lower) semi-continuous at $T$, provided that if $T_{n} \rightarrow T$ (in the norm topology) then $\lim \sup f\left(T_{n}\right) \subset f(T)$ $\left(f(T) \subset \liminf f\left(T_{n}\right)\right)$. If $f$ is both upper and lower semi-continuous at $T$, then it is said to be continuous at $T$ and in this case $\lim f\left(T_{n}\right)=f(T)$.

2. Main results. When $A, A_{n} \in B(H)$ and $B, B_{n} \in B(K)$ are given we denote by $M_{C}$ and $M_{n}$ the operators acting on $H \oplus K$ defined by

$$
M_{C}=\left(\begin{array}{cc}
A & C \\
0 & B
\end{array}\right), M_{n}=\left(\begin{array}{cc}
A_{n} & C_{n} \\
0 & B_{n}
\end{array}\right),
$$

where $C, C_{n} \in B(K, H)$.

Consider the following example: let $U \in B\left(l_{2}\right)$ be the unilateral shift, $A_{n}=U$, $B_{n}=U^{*}$, and $C_{n}=\frac{1}{n}\left(I-U U^{*}\right)$. Then on $l_{2} \oplus l_{2}$ we have

$$
M_{n}=\left(\begin{array}{cc}
A_{n} & \frac{1}{n}\left(I-U U^{*}\right) \\
0 & B_{n}
\end{array}\right) \rightarrow M=\left(\begin{array}{cc}
U & 0 \\
0 & U^{*}
\end{array}\right),
$$

as $n \longrightarrow \infty$. For operator matrices $M_{n}$ and $M$ we have $\sigma\left(M_{n}\right)=\{\lambda \in \mathbb{C}:|\lambda|=1\}$ and $\sigma(M)=\{\lambda \in \mathbb{C}:|\lambda| \leq 1\}$. Therefore $\sigma\left(M_{n}\right) \not \rightarrow \sigma\left(M_{n}\right)$.

However, we have the following result.

Theorem 2.1. Let $A \in B(H)$ and $B \in B(K)$ be such that $\sigma(A) \cap \sigma(B)=\emptyset$. Then $\sigma$ is continuous at $A$ and $B$ if and only if $\sigma$ is continuous at $M_{C}$, for every $C \in B(K, H)$.

Proof. Since $\sigma(A) \cap \sigma(B)=\emptyset$, there exists $\delta>0$ such that $d(\sigma(A), \sigma(B))>3 \delta$. Now, by the upper semi-continuity of the spectrum at $A$ and $B$ [11], for every sequence $\left(A_{n}\right)$ in $B(H)$ and every sequence $\left(B_{n}\right)$ in $B(K)$ such that $A_{n} \rightarrow A$ and $B_{n} \rightarrow B$ there exists a natural number $n_{0}$ such that $n \geq n_{0} \Rightarrow \sigma\left(A_{n}\right) \subset(\sigma(A))_{\delta}$ and $\sigma\left(B_{n}\right) \subset(\sigma(B))_{\delta}$. Since $\sigma\left(A_{n}\right) \cap \sigma\left(B_{n}\right)=\emptyset$, for every $n \geq n_{0}$, we have that $\sigma\left(M_{n}\right)=$ $\sigma\left(A_{n}\right) \cup \sigma\left(B_{n}\right)$.

$(\Rightarrow)$ Suppose that $\sigma$ is continuous at $A$ and $B$. Then

$$
\sigma\left(M_{c}\right)=\sigma(A) \cup \sigma(B) \subset \liminf \left(\sigma\left(A_{n}\right) \cup \sigma\left(B_{n}\right)\right)=\lim \inf \sigma\left(M_{n}\right) .
$$

Therefore $\sigma$ is lower semi-continuous at $M_{C}$, and hence $\sigma$ is continuous at $M_{C}$ for every $C \in B(K, H)$. 
$(\Leftarrow)$ Suppose that $\sigma$ is continuous at $M_{C}$ for every $C \in B(K, H)$. We shall show that $\sigma$ is continuous at $A$. Let $\lambda \in \sigma(A)$. Then $\lambda \notin \sigma(B)$ and

$$
\lambda \in \sigma(A) \subset \sigma\left(M_{C}\right) \subset \liminf \sigma\left(M_{n}\right) .
$$

Therefore there exists a sequence $\left(\lambda_{n}\right)$ such that $\lambda_{n} \in \sigma\left(M_{n}\right)$ and $\lambda_{n} \rightarrow \lambda$. But $\sigma\left(A_{n}\right) \cap \sigma\left(B_{n}\right)=\emptyset$, for every $n \geq n_{0}$; hence we have $\sigma\left(M_{n}\right)=\sigma\left(A_{n}\right) \cup \sigma\left(B_{n}\right)$. If there exists a subsequence $\left(\lambda_{n_{k}}\right)$ of $\left(\lambda_{n}\right)$ such that $\lambda_{n_{k}} \in \sigma\left(B_{n_{k}}\right)$, then we have $\lambda \in \lim \sup \sigma\left(B_{n}\right) \subset \sigma(B)$. This is a contradiction. Therefore $\lambda_{n} \in \sigma\left(A_{n}\right)$, for every $n \geq n_{0}$. Thus $\lambda \in \liminf \sigma\left(A_{n}\right)$, and hence $\sigma$ is continuous at $A$. Similarly, $\sigma$ is continuous at $B$.

If $A \in B(H)$ and $B \in B(K)$ such that $\omega(A) \cap \omega(B)=\emptyset$, then we have $\omega\left(M_{C}\right)=\omega(A) \cup \omega(B)[\mathbf{1 0}$, Theorem 4]. Now, we have the following theorem.

Theorem 2.2. Let $A \in B(H)$ and $B \in B(K)$ such that $\omega(A) \cap \omega(B)=\emptyset$. Then $\omega$ is continuous at $A$ and $B$ if and only if $\omega$ is continuous at $M_{C}$, for every $C \in B(K, H)$. 2.1 .

Proof. Since $\omega$ is upper semi-continuous, the proof is similar to that of Theorem

$\alpha$ and $\beta$ can be viewed as functions assigning $\alpha(T)$ and $\beta(T)$ to each $T \in B(H)$, respectively.

Theorem 2.3. Let $A \in B(H), B \in B(K)$ and $C \in B(K, H)$ such that

(1) $\sigma\left(M_{C}\right)=\sigma_{a}(A) \cup \sigma_{d}(B) \cup\{\lambda \in \mathbb{C} ; \alpha(B-\lambda) \neq \beta(A-\lambda)\}$;

(2) $\sigma_{a}$ is continuous at $A$;

(3) $\sigma_{d}$ is continuous at $B$.

Then $\sigma$ is continuous at $M_{C}$.

Proof. It is sufficient to show that $\sigma$ is lower semi-continuous at $M_{C}$. Let $\lambda \in \sigma\left(M_{C}\right)$. We shall divide the proof into three cases.

Case 1. If $\lambda \in \sigma_{a}(A)$, then since $\sigma_{a}$ is continuous at $A$ there exists a natural number $n_{0}$ such that for every $n>n_{0}$ we have $\lambda \in \sigma_{a}\left(A_{n}\right) \subset \sigma\left(M_{n}\right)$.

Case 2. If $\lambda \in \sigma_{d}(B)$, then by continuity of $\sigma_{d}$ at $B$ there exists a natural number $n_{1}$ such that for every $n>n_{1}$ we have $\lambda \in \sigma_{d}\left(B_{n}\right) \subset \sigma\left(M_{n}\right)$.

Case 3. Suppose that $\lambda \in \sigma\left(M_{C}\right) \backslash\left(\sigma_{a}(A) \cup \sigma_{d}(B)\right)$. Then we have $\alpha(B-\lambda) \neq$ $\beta(A-\lambda), \alpha(A-\lambda)=0$, and $\beta(B-\lambda)=0$. Therefore $i(A-\lambda) \neq i(B-\lambda)$, and hence it follows from the continuity of the index that there exists $n_{2}$ such that for $n>n_{2}$, $i\left(A_{n}-\lambda\right) \neq i\left(B_{n}-\lambda\right)$. Since functions $\alpha$ and $\beta$ are continuous at $A$ and $B[3$, Corollary 2.3], respectively, we have that $\alpha\left(B_{n}-\lambda\right) \neq \beta\left(A_{n}-\lambda\right)$. Therefore $\lambda \in \sigma\left(M_{n}\right)$ for every $n>n_{2}$. It follows that in all three cases $\sigma$ is continuous at $M_{C}$.

If $M_{C}$ obeys Browder's theorem, then the Weyl spectrum, the Browder spectrum and the spectrum are continuous at $M_{C}$.

Theorem 2.4. Let $A \in B(H), B \in B(K)$ and $C \in B(K, H)$ such that

(1) $\sigma\left(M_{C}\right)$ obeys Browder's theorem;

(2) $\sigma_{a}$ is continuous at $A$; 
(3) $\sigma_{d}$ is continuous at B.

Then $\sigma, \omega$, and $\sigma_{b}$ are continuous at $M_{C}$, respectively.

Proof. Let $\lambda \in \sigma\left(M_{C}\right)$. If $\lambda \in \sigma_{a}(A) \cup \sigma_{d}(B) \cup\{\lambda \in \mathbb{C} ; \alpha(B-\lambda) \neq \beta(A-\lambda)\}$, then it follows from Theorem 2.3 that $\lambda \in \lim \inf \sigma\left(M_{n}\right)$. Suppose now that

$$
\lambda \in \sigma\left(M_{C}\right) \backslash\left[\sigma_{a}(A) \cup \sigma_{d}(B) \cup\{\lambda \in \mathbb{C} ; \alpha(B-\lambda) \neq \beta(A-\lambda)\}\right] .
$$

Then $\alpha(A-\lambda)=\beta(B-\lambda)=0, \alpha(B-\lambda)=\beta(A-\lambda)$, and so $i(A-\lambda)=-i(B-\lambda)$. By [1, Lemma 1.2], $i\left(M_{C}-\lambda\right)=0$. Since $M_{C}$ obeys Browder's theorem, $\lambda \notin \sigma_{b}\left(M_{C}\right)$. Therefore $\lambda$ is an isolated point of $\sigma\left(M_{C}\right)$, and so $\lambda \in \lim \inf \sigma\left(M_{n}\right)$. Hence $\sigma$ is continuous at $M_{C}$. It follows from [2, Theorem 2.2] that $\omega$ and $\sigma_{b}$ are continuous at $M_{C}$.

\section{REFERENCES}

1. J. B. Conway and B. B. Morrel, Operators that are points of spectral continuity II, Integral Equations and Operator Theory 4 (1981), 459-503.

2. S. V. Djordjević and Y. M. Han, Browder's theorems and spectral continuity, Glasgow Math. J. 42 (2000), 479-486.

3. Fernando Galaz-Fantes, Approximation by semi-Fredholm operators, Proc. Amer. Math. Soc. 120 (1994), 1219-1222.

4. J. K. Han, H. Y. Lee and W. Y. Lee, Invertible completions of $2 \times 2$ upper triangular operator matrices, Proc. Amer. Math. Soc. 128 (1999), 119-123.

5. R. E. Harte, Invertibility and singularity for bounded linear operators (Marcel Dekker, New York, 1988).

6. R. E. Harte and W. Y. Lee, Another note on Weyl's theorem, Trans. Amer. Math. Soc. 349 (1997), 2115-2124.

7. Du Hong-Ke and Pan Jin, Perturbation spectrum of $2 \times 2$ operator matrices, Acta Sci. Math. (Szeged) 64 (1998), 259-269.

8. T. Kato, Perturbation theory of linear operators (Springer-Verlag, 1976).

9. W. Y. Lee, Weyl's theorem for operator matrices, Integral Equations Operator Theory 32 (1998), 319-331.

10. W. Y. Lee, Weyl spectra of operator matrices, Proc. Amer. Math. Soc. 129 (2001), $131-138$.

11. J. D. Newburgh, The variation of spectra, Duke Math. J. 18 (1951), 166-176. 